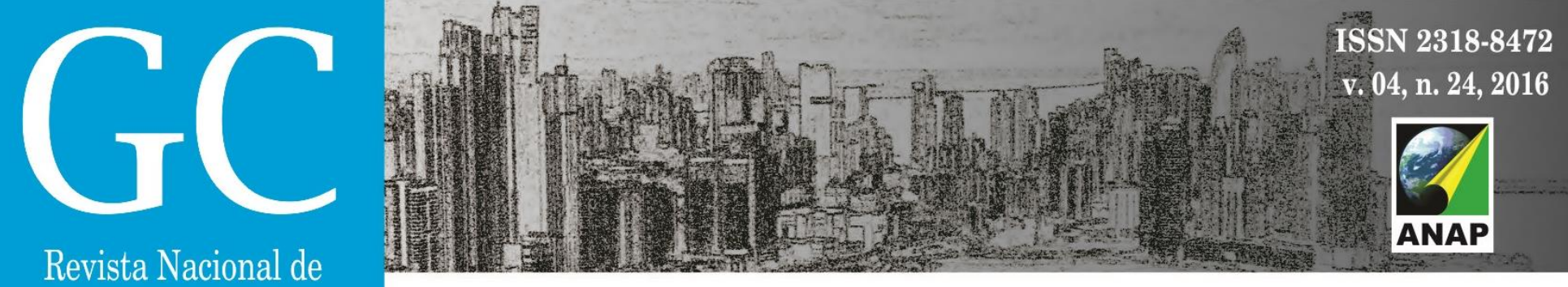

Gerenciamento de Cidades

National Journal of Cities Management

\title{
Avaliação da eficiência do tratamento de efluentes em um abatedouro do município de Passos-MG
}

Wastewater treatment efficiency evaluation in a slaughterhouse in the city of Passos-MG

Evaluación de la eficiencia tratamiento de aguas residuales en un matadero en la ciudad de Passos-MG

\author{
Caroline da Silva Oliveira \\ Mestranda, UNESP, Brasil. \\ oliveira.caroline.dasilva@gmail.com
}

Paula Nunes Coelho Doutoranda, UNESP, Brasil. paulinhancoelho@gmail.com

Odila Rigolin de Sá

Professora Doutora, UEMG, Brasil. odilarigolin@yahoo.com.br 


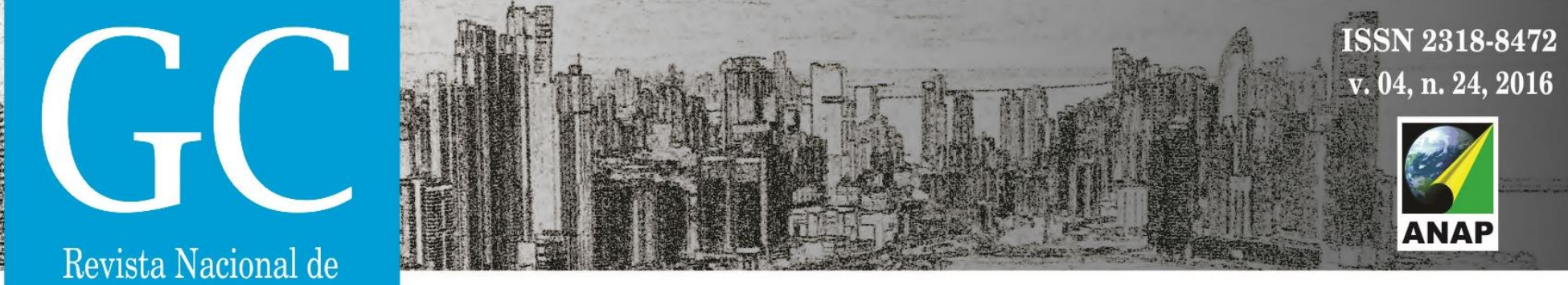
Gerenciamento de Cidades

\section{RESUMO}

O crescimento da população e a aceleração da economia ampliam os usos múltiplos pela água resultando em um aumento na produção de efluentes. A indústria alimentícia de carne é responsável em produzir grande volume de resíduos líquidos com alto potencial poluidor. $O$ tratamento de efluentes é uma alternativa para evitar prejuízos ambientais. Neste trabalho avaliamos a eficiência do tratamento de efluente em um abatedouro no município de Passos- MG. Foram realizadas análises físicas e químicas do efluente em cada etapa do tratamento (lagoa anaeróbia, facultativa e aerada) e os valores obtidos foram comparados com a Resolução CONAMA no 430/2011. O tratamento do abatedouro foi eficiente na remoção de matéria orgânica, demanda bioquímica de oxigênio, demanda química de oxigênio e materiais sedimentáveis. Para o nitrogênio, o tratamento não foi eficiente, demostrando a necessidade de uma revisão no funcionamento do sistema, evitando prejuízos a saúde dos seres vivos e ao meio ambiente.

PALAVRAS-CHAVE: Água Residuária. Resolução CONAMA 430. Meio Ambiente.

\section{ABSTRACT}

The increasing population and economic acceleration expand multiple uses by water resulting in an increase in the production of effluents. The food meat industry is responsible for producing large volumes of liquid waste with a high pollution potential. The wastewater treatment is an alternative to avoid environmental damage. In this work we evaluate the effectiveness in treatment of effluent from a slaughterhouse in the city of Passos - MG. Physical and chemical analysis of the effluent were carried out at each stage of treatment (anaerobic lagoon, facultative and aerated). The values obtained were compared with the CONAMA Resolution $\mathrm{n}$ - 430/2011. Treatment of slaughterhouse was efficient in removing organic matter, biochemical oxygen demand, chemical oxygen demand and sedimentary materials. The treatment was not effective for nitrogen, demonstrating the need for a revision in the system operation, preventing damage to the health of living beings and the environment.

KEYWORDS: Wastewater. Resolution CONAMA 430. Environment.

\section{RESUMEN}

Crecimiento de la población y la aceleración económica amplifican múltiples usos por el agua que resulta en un aumento en la producción de efluentes. La industria de la carne de alimentos es responsable de producir grandes volúmenes de residuos líquidos con un alto potencial de contaminación. El tratamiento de aguas residuales es una alternativa para evitar daños ambientales. En este trabajo se evalúa la eficacia de tratamiento de efluentes en un matadero en la ciudad de Passos-MG. El análisis físico y químico del efluente se llevaron a cabo en cada etapa del tratamiento (laguna anaeróbica, facultativa y aireado) y los valores obtenidos se compararon con la Resolución CONAMA no 430/2011. El tratamiento del matadero fue eficiente en la eliminación de la materia orgánica, demanda bioquímica de oxígeno, demanda química de oxígeno y materiales sedimentários. Para el nitrógeno, el tratamiento no fue efectivo, lo que demuestra la necesidad de una revisión en el funcionamiento del sistema, la prevención de daños a la salud de los seres vivos y el medio ambiente.

PALABRAS CLAVE: Las Aguas Residuales. Resolución CONAMA 430. Medio ambiente. 


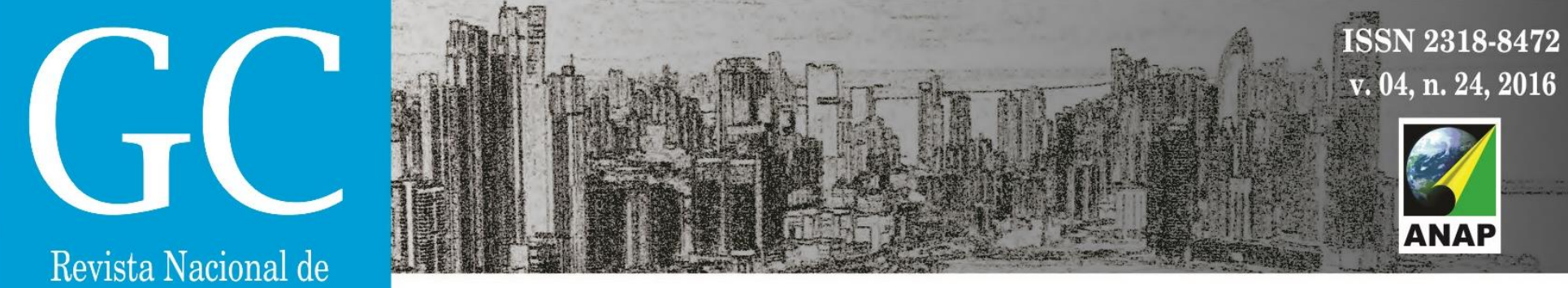

Gerenciamento de Cidades

fornecimento de energia elétrica e adequada eficiência na remoção de diversas categorias de poluentes (matéria orgânica biodegradável, nutrientes, agentes patógenos e sólidos suspensos) (CHERNICHARO, 1997).

Considerando a importância da escassez hídrica e dos impactos ambientais gerados durante o processamento da carne em abatedouro, o objetivo do estudo foi avaliar a eficiência do tratamento de efluentes em um abatedouro do município de Passos-MG e verificar se o lançamento dos efluentes tratados encontra-se em conformidade com a Resolução CONAMA $n^{\circ} 430 / 2011$.

\section{MATERIAL E MÉTODOS}

O estudo foi desenvolvido na estação de tratamento de efluentes, presente em um abatedouro bovino e suíno, situado no município de Passos, estado de Minas Gerais (Figura 1).

Figura 1. Imagem de satélite do sistema de tratamento de efluente (Lagoas de estabilização) do abatedouro em Passos-MG.

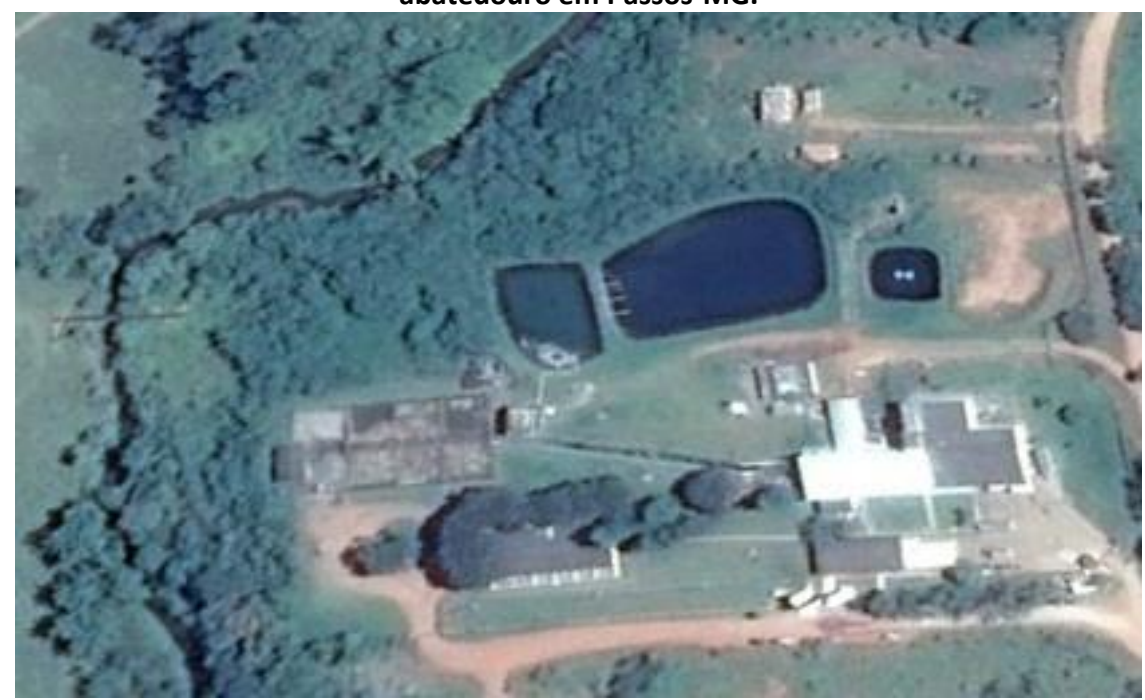

Fonte: GOOGLE EARTH, 2014.

O abatedouro possui um abate médio de 700 bois e 1200 suínos por semana. Conforme a Deliberação Normativa (DN) $n^{\circ} 74$ do Conselho Estadual de Política Ambiental (COPAM-MG) o abatedouro é considerado um empreendimento de porte médio, com potencial poluidor/degradador grande. Seu sistema de tratamento é constituído de tratamento preliminar, primário e secundário (Figura 2).

O tratamento preliminar se inicia na separação de sólidos grosseiros por meio da caixa de separação de resíduos. Os resíduos mais grosseiros são utilizados para adubo de capim ou produção de ração, evitando uma quantidade muito elevada da demanda bioquímica de oxigênio (DBO) no efluente. 


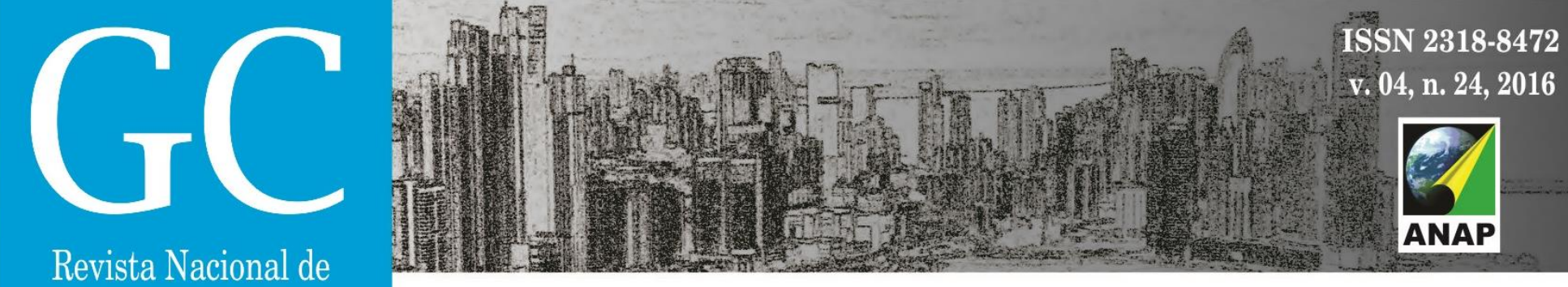

Gerenciamento de Cidades

O primário visa á remoção de sólidos em suspensão não grosseiros por decantação e implica na redução da carga de DBO dirigida ao tratamento secundário. O tratamento secundário consiste na remoção de material orgânico fino em suspensão, não removido no tratamento preliminar e primário, decorrentes das transformações bioquímicas, produzidas pelos microrganismos (lagoa anaeróbia, facultativa e aerada). Em seguida, o efluente é transferido para o medidor de vazão (efluente tratado), que mede a quantidade de esgoto a ser despejado e posteriormente lançado no corpo hídrico Ribeirão Bocaina (Figura 2).

Figura 2. Fluxograma geral da estação de tratamento de efluentes (ETE) do abatedouro.

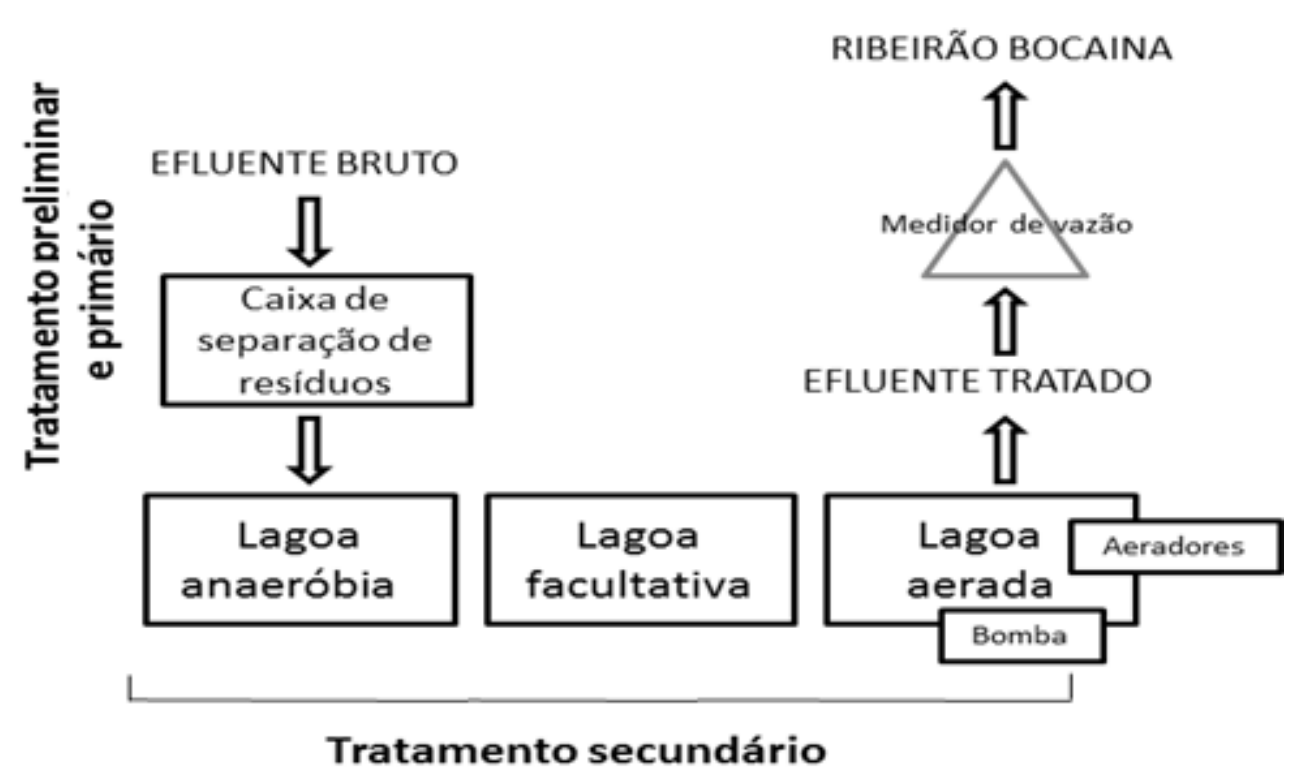

Fonte: DO AUTOR, 2016.

A eficiência no tratamento foi realizada a partir do monitoramento das variáveis físicas e químicas dos efluentes coletados nos meses de agosto e setembro de 2014. Foram coletados em quatro pontos, sendo: efluente bruto sem tratamento (Figura 3A), saída lagoa anaeróbia e entrada lagoa facultativa (Figura 3B), facultativa (Figura 3C), aerada (Figura 3D).

A temperatura e o valores do $\mathrm{pH}$ da água foram obtidas com um $\mathrm{pH}$-metro digital. $\mathrm{A}$ concentração de oxigênio dissolvido foi realizada pelo aparelho oxímetro Datalogger (ITT 71440). Para analisar a demanda bioquímica de oxigênio (DBO) foi utilizado o método da diluição e incubação pela ação de bactérias, por 5 dias a $20^{\circ} \mathrm{C}$, medida pelo sensor Oxitop (ABNT, 1992). A demanda química de oxigênio (DQO) foi determinada pelo aparelho Bloco Digestor e a leitura realizada pelo Colorimeter (APHA 2012). A concentração de material sedimentável foi obtida pela decantação do sedimento (NBR/88), adicionando um litro do 
Revista Nacional de Gerenciamento de Cidades

BUSTILLO-LECOMPTE, C. F.; MEHRVAR, M. Slaughterhouse wastewater characteristics, treatment, and management in the meat processing industry: A review on trends and advances. Journal of environmental management, v. 161, p. 287-302, 2015.

CHERNICHARO, C. A. L. Princípios do tratamento biológico de águas residuárias - Volume 5: Reatores anaeróbios. Departamento de Engenharia Sanitária e Ambiental - UFMG. Belo Horizonte, 245 p.,1997.

CONSELHO NACIONAL DO MEIO AMBIENTE - CONAMA. 2011. Resolução CONAMA n430. Disponível em: http://www.mma.gov.br/port/conama/legiabre.cfm?codlegi=646. Acessado em 30 de agosto de 2016.

Deliberação Normativa COPAM no 74 , de 9 de setembro de 2004. Disponível em: http://sisemanet.meioambiente.mg.gov.br/mbpo/recursos/DeliberaNormativa74.pdf. Acessado em 09/09/2016.

DORGHAM, M. M. Effects of eutrophication. In: ASARI, A. A.; GILL, S. S. (eds.). Eutrophication: Causes, Consequences and Control, v. 2, p. 29-44, 2014.

ESTEVES, F. A. Fundamentos de limnologia. 3. ed. Rio de Janeiro: Interciências, 2011.

GOLTERMAN, H. L.; CLYMO, R. S.; OHNSTAD, M. A. M. Methods for physical and chemical analysis of freshwaters. 2a ed. Oxford: Blackwell Scientific Publications, 1978. 213 p.

HESPANHOL, I. Um novo paradigma para a gestão de recursos hídricos. Estudos Avançados, v. 22, n. 63, p. 131$158,2008$.

JORDAO, P. E.; PESSOA, C. A. Tratamento de esgotos domésticos. Rio de Janeiro: ABES, v. 6, 2011. 1087 p.

LIMA, F. P. Energia no tratamento de esgoto: Análise tecnológica e institucional para conservação de energia e uso de biogás. Dissertação de mestrado. Programa Inter unidades de Pós-Graduação em Energia da Universidade de São Paulo. São Paulo, 2005.

(NBR 10561/88- Água - Determinação de resíduo sedimentável (sólidos sedimentáveis) - Método do cone de Imhoff) - Método de ensaio.

OPEN Reflux method. In: STANDARD methods for the examination of water and wastewater. 22nd ed. Washington: APHA; AWWA; WEF, 2012. p. 5:17-18 (Method 5220 B).

RODRIGUES, L. S.; LOPES, B. C.; LIMA, C. A.; RIBEIRO, M. C.; SANTOS, R. P.; SILVA, I. J. Tratamento de efluentes de abatedouro de frangos por meio de reator UASB seguido de filtro anaeróbio. Arq. bras. med. vet. zootec, v. 68, n. 1, p. 97-103, 2016

Secretaria Nacional de Saneamento Ambiental - SNSA. Sistema Nacional de Informações sobre Saneamento: Diagnóstico dos Serviços de Água e Esgotos - 2014. Brasília: SNSA/MCIDADES, 2016. 212 p.

SMITH, V. H.; SCHINDLER, D. W. Eutrophication science: where do we go from here? Trends in Ecology and Evolution, v.24, n.4, 2009.

SPERLING, M. V. Princípios do Tratamento Biológico de Águas Residuárias, Universidade Federal de Minas Gerais: 2002 - Volume 1, 3 e 4.

TAVARES, E. M; WEBER, M. I. Impactos ambientais e tratamento gerado pelos efluentes de abatedouro bovinos, 2012. Universidade Federal do Paraná, 2012.

VALDERRAMA, J. C. The simultaneous analysis of total nitrogen and total phosphorus in natural waters. Marine 


\section{Revista Nacional de}

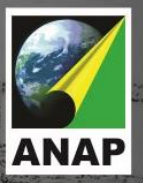

\section{Gerenciamento de Cidades}

chemistry, v. 10, n. 2, p. 109-122, 1981.

VON SPERLING, M. Princípios do tratamento biológico de águas residuárias - Volume 1: Introdução à qualidade das águas e ao tratamento de esgotos. Departamento de Engenharia Sanitária e Ambiental - UFMG. Belo Horizonte, 240 p., 1995.

\section{ANEXOS}

Tabela 3. Valores dos parâmetros físicos e químicos avaliados em efluentes de abatedouro obtidas nas lagoas de tratamento, no mês de agosto de 2014. M.S: materiais sedimentáveis; MO: matéria orgânica; DQO: demanda química de oxigênio; DBO: demanda bioquímica de oxigênio.

\begin{tabular}{lccccccc}
\hline \multicolumn{1}{c}{ Parâmetros } & $\mathrm{pH}$ & $\begin{array}{c}\text { Temperatura } \\
\left({ }^{\circ} \mathrm{C}\right)\end{array}$ & $\begin{array}{c}\mathrm{M} . \mathbf{S} \\
(\mathrm{ml} / \mathrm{L})\end{array}$ & $\begin{array}{c}\text { M.O } \\
(\mathbf{m g} / \mathrm{L})\end{array}$ & $\begin{array}{c}\text { DQO } \\
(\mathbf{m g} / \mathrm{L})\end{array}$ & $\begin{array}{c}\text { DBO } \\
(\mathbf{m g} / \mathrm{L})\end{array}$ & $\begin{array}{c}\text { Nitrogênio } \\
(\mathbf{m g} / \mathrm{L})\end{array}$ \\
\hline $\begin{array}{l}\text { Efluente bruto } \\
\begin{array}{l}\text { Entrada lagoa } \\
\text { facultativa }\end{array}\end{array}$ & 6,65 & 23,4 & 250 & 420 & 1650 & 1455 & 184,8 \\
$\begin{array}{l}\text { Entrada lagoa } \\
\text { aerada }\end{array}$ & 7,52 & 22,3 & 5 & 84 & 477 & 203 & 172,2 \\
Efluente tratado & 7,78 & 22,3 & 1 & 84 & 458 & 124 & 159,6 \\
\hline
\end{tabular}

Fonte: DO AUTOR, 2016.

Tabela 4. Valores dos parâmetros físicos e químicos avaliados em efluentes de abatedouro obtidas nas lagoas de tratamento, no mês de setembro de 2014. M.S: materiais sedimentáveis; MO: matéria orgânica; DQO: demanda química de oxigênio; DBO: demanda bioquímica de oxigênio.

\begin{tabular}{|c|c|c|c|c|c|c|c|}
\hline Parâmetros & $\mathrm{pH}$ & $\begin{array}{c}\text { Temperatura } \\
\left({ }^{\circ} \mathrm{C}\right)\end{array}$ & $\begin{array}{c}\text { M. S } \\
\text { (ml/L) }\end{array}$ & $\begin{array}{c}\mathrm{M} .0 \\
(\mathrm{mg} / \mathrm{L})\end{array}$ & $\begin{array}{c}\mathrm{DQO} \\
(\mathrm{mg} / \mathrm{L})\end{array}$ & $\begin{array}{c}\text { DBO } \\
(\mathrm{mg} / \mathrm{L})\end{array}$ & $\begin{array}{c}\text { Nitrogênio } \\
(\mathrm{mg} / \mathrm{L})\end{array}$ \\
\hline Efluente bruto & 6,86 & 20 & 190 & 510 & 1650 & 1596 & 352,8 \\
\hline $\begin{array}{l}\text { Entrada lagoa } \\
\text { facultativa }\end{array}$ & 6,89 & 20 & 10 & 88 & 609 & 163 & 176,4 \\
\hline $\begin{array}{l}\text { Entrada lagoa } \\
\text { aerada }\end{array}$ & 7,73 & 20 & 1 & 84 & 492 & 95,8 & 172,2 \\
\hline Efluente tratado & 7,86 & 22,6 & 1 & 84 & 427 & 73,2 & 149,8 \\
\hline
\end{tabular}

Fonte: DO AUTOR, 2016. 